\title{
Medikamentensicherheit bei Kindernotfällen - dringend notwendig und einfach umsetzbar: die Empfehlungen der neuen AWMF-S2k-Leitlinie
}

Alle Arbeiten, die sich damit befassen, finden erschreckend hohe Inzidenzen von Medikationsfehlern bei Kindernotfällen oder in entsprechenden Simulationsszenarien [1]. Dies liegt vor allem daran, dass bei Kindern eine individuell zu berechnende, gewichtsbezogene Dosis gegeben werden muss. Dies steht in deutlichem Kontrast beispielsweise zur Reanimation von Erwachsenen, die leitliniengerecht unabhängig von Alter oder Gewicht immer 1mg Adrenalin erhalten. Zusätzlich stellt die Notfallversorgung eines Kindes oft eine Situation extremer Belastung für den Verordnenden dar. Beispielsweise waren die Dosierungen von Adrenalin in den einzigen beiden Studien, die dies im tatsächlichen prähospitalen Alltag untersuchten, in $60 \%$ [2] bzw. 100\% [3] der Fälle nicht korrekt. Die durchschnittliche Überdosierung lag bei 808 bzw. $882 \%$ der empfohlenen Dosis und somit nah an $1000 \%$, was einem mit dem Überleben kaum zu vereinbarenden 10er-Potenz-Fehler entspricht. Diese Feststellungen sollen keinesfalls anklagen, sondern nur wachrütteln. Und alleine in der Erhöhung der Vigilanz, also in dem Erkennen der Bedeutsamkeit der Medikationssicherheit und der eigenen Fehlbarkeit, liegen die wichtigsten Voraussetzungen für eine Verbesserung [4].

Denn einfach umzusetzende Maßnahmen können die Medikamentensicherheit bei Kindernotfällen erheblich verbessern. Dieses Ziel hat sich die gleichnamige AWMF-Leitlinie gesetzt und mit Beteiligung von 15 Fachgesellschaften, Berufsverbänden und Interessenverbänden folgende wesentliche Empfehlungen zusammengestellt [5] und mit 100\%igem, formal von der AWMF moderierten Konsens beschlossen.

- Ein Medikament, zu welchem dem Anwender hinreichende pharmakologische Kenntnis für die Notfallindikation fehlt, soll nicht verabreicht werden („primum non nocere“).

- Vor jeder Medikamentengabe soll im 4-Augen-Prinzip überprüft werden, dass es sich um das richtige Medikament, in der richtigen Dosis, zum richtigen Zeitpunkt, mit dem richtigen Verabreichungsweg und für den richtigen
Patient handelt (Anwenden der 5-R-Regel).

- Vor jeder Therapie soll die Indikation hinterfragt und geprüft werden.

- Medikamente mit geringem Verteilungsvolumen und geringer therapeutischer Breite sollen bei deutlicher Adipositas am Normalgewicht ausgerichtet dosiert werden.

- Eine „Übertherapie“ soll vermieden werden (so wenig wie möglich und so viel wie nötig).

- Die Verordnung von Notfallmedikamenten soll unter Kenntnis und Verwendung pädiatrisch-pharmakologischer Referenzen bzw. kognitiver Hilfsmittel erfolgen.

- Vor jeder medikamentösen Therapie soll das Gewicht des Kindes ermittelt und dokumentiert werden.

- Wenn kein bekanntes Gewicht verfügbar ist, soll eine längenbezogene Gewichtsschätzung durchgeführt werden.

- Die Gaben von Medikamenten, die eine geringe therapeutische Breite aufweisen oder bei Fehldosierung großen Schaden anrichten können (z.B. Adrenalin, Analgetika), sollen NICHT ohne vorherige Überprüfung durch ein unterstützendes System (z.B. Tabelle, Lineale) erfolgen.

- Längenbezogene Systeme zur Gewichtsschätzung mit Dosisempfehlung sollten vor allem prähospital bevorzugt eingesetzt werden.

- Mündliche Verordnungen sollen eine klare Struktur haben, eindeutig und vollständig sein sowie schnellstmöglich schriftlich dokumentiert werden; wenn immer möglich sollen Verordnungen primär schriftlich erfolgen.

- Jede Verordnung soll durch alle Beteiligten laut wiederholt und bestätigt werden.

- Jede aufgezogene Spritze soll vorzugsweise mit einem Etikett nach ISO 26852 längs so beklebt werden, dass die Skalierung weiter lesbar bleibt.

- Die nicht technischen Fähigkeiten und die Inhalte von Versorgungsstandards sollen trainiert werden (z.B. Simulationstraining).
Zusätzlich bietet die S2k-Leitlinie unter Freigabe der Präsidien aller beteiligten Gesellschaften erstmalig im deutschsprachigen Raum eine klare Positionierung zum „Off-Label-Use“ in der pädiatrischen Notfallmedizin. „Ein ,Off-Label-Use“ ist nicht unsachgemäß, illegal oder kontraindiziert, sondern kann die bestmögliche Therapie darstellen. Ein grundsätzlicher Verzicht auf einen ,Off-Label-Use' gefährdet Kinder und macht eine sachgemäße Behandlung unmöglich“, heißt es darin. Die prähospitale und innerklinische Behandlung von Notfällen bei Kindern sollte durch Therapieentscheidungen gelenkt werden, die auf wissenschaftlicher Evidenz und Erfahrung basieren und nicht allein aufgrund des Zulassungsstatus erfolgen. Im Zentrum dieses Abschnitts der Leitlinie steht eine Tabelle mit dem Einschluss von „Off-Label-Medikamenten“ für den Kindernotfall. Diese Liste, die vor allem von Prof. Dr. Wolfgang Rascher und Prof. Dr. Antje Neubert (beide in der DGKJ-Arzneimittelkommission) erarbeitet wurde, zeigt Dosierungsempfehlungen inklusive der zugrunde liegenden Referenzen für die genannten Empfehlungen. Damit kann sie zu einer zentralen pharmakologischen Referenz bei Kindernotfällen werden und bietet Ärztinnen und Ärzten eine klare und verlässliche Absicherung für die Medikamentengabe.

Interessenkonflikt

Priv.-Doz. Dr. Jost Kaufmann hält ein europaweites Patent für die Entwicklung des Pädiatrischen Notfalllineals (PädNFL; www.notfalllineal.de), verzichtet aber auf jegliche Vergütungen für dessen Vermarktung. Die anderen Autoren berichten keinerlei Interessenkonflikt.

\section{Autorinnen/Autoren}

\section{Priv.-Doz. Dr. Jost Kaufmann ${ }^{1}$}

1 Abteilung für Kinderanästhesie, Kinderkrankenhaus der Kliniken der Stadt Köln gGmbH 


\section{Korrespondenzadresse}

Priv.-Doz. Dr. Jost Kaufmann

Abteilung für Kinderanästhesie

Kinderkrankenhaus der Kliniken

der Stadt Köln gGmbH

Amsterdamer Str. 59

50735 Köln

Deutschland

jost.kaufmann@uni-wh.de

Literatur

[1] Kaufmann J, Laschat M, Wappler F. Medication errors in pediatric emergencies: a systematic analysis. Dtsch Arztebl Int 2012; 109: 609-616
[2] Hoyle JD, Davis AT, Putman KK et al. Medication dosing errors in pediatric patients treated by emergency medical services. Prehosp Emerg Care 2012; 16: 59-66

[3] Kaufmann J, Roth B, Engelhardt T et al. Development and Prospective Federal StateWide Evaluation of a Device for Height-Based Dose Recommendations in Prehospital Pediatric Emergencies: A Simple Tool to Prevent Most Severe Drug Errors. Prehosp Emerg Care 2018; 22: 252-259

[4] Kaufmann J, Schieren M, Wappler F. Medication errors in paediatric anaesthesia-a cultural change is urgently needed!. $\mathrm{Br}$ J Anaesth 2018; 120: 601-603

[5] Arbeitsgemeinschaft der Wissenschaftlichen Medizinischen Fachgesellschaften e.V. (AWMF). S2k - Leitlinie 027/071 „Medikamentensicherheit bei Kindernotfällen“.
2021. Im Internet (Stand: 26.04.2021): https://www.awmf.org/leitlinien/detail/II/ 027-071.html

Bibliografie

Notarzt 2021; 37: 149-150

online publiziert 12.05.2021

DOI 10.1055/a-1468-1343

ISSN 0177-2309

(c) 2021. Thieme. All rights reserved. Georg Thieme Verlag KG, Rüdigerstraße 14, 70469 Stuttgart, Germany 